\title{
Alimentação e nutrição no contexto da atenção básica e da promoção da saúde: a importância de um diálogo
}

\section{Feeding and nutrition in primary health care and health promotion: the importance of a dialogue}

\author{
Viviane Rangel de Muros Pimentel ${ }^{1}$ \\ Maria Fátima de Sousa ${ }^{2}$ \\ Luciani Martins Ricardi ${ }^{3}$ \\ Edgar Merchan Hamann² \\ 1 Núcleo de Estudos de Saúde Pública - CEAM/ \\ UnB. Universidade de Brasília. Brasília-DF, Brasil. \\ ${ }^{2}$ Departamento de Saúde Coletiva, Faculdade \\ de Ciências da Saúde, Universidade de Brasília. \\ Brasilia-DF, Brasil. \\ ${ }^{3}$ Curso de Pós-graduação em Saúde Coletiva, \\ Universidade de Brasília. Brasília-DF, Brasil. \\ Agente financiador: Ministério da Ciência, \\ Tecnologia e Inovação (MCTI). Número de \\ processo: $2008 \mathrm{NCO00162.}$ \\ Correspondência / Correspondence \\ Luciani Martins Ricardi3 \\ E-mail: luciani_snp@hotmail.com
}

\section{Resumo}

A transição nutricional é um dos maiores desafios para as políticas públicas de saúde, pois demanda um modelo de atenção pautado na integralidade e uma abordagem voltada à promoção da saúde, em especial na Atenção Básica. A alimentação e a nutrição são requisitos básicos para a promoção da saúde e permitem alcançar o potencial de desenvolvimento humano. O objetivo deste artigo foi refletir sobre os campos da Alimentação e Nutrição no contexto da Atenção Básica e da Promoção da Saúde, por meio da metodologia descritivo-reflexiva, reforçando a necessidade de um diálogo entre essas áreas, por meio da interlocução entre seus desafios e potencialidades, para que sejam alcançados os princípios do Sistema Único de Saúde. A análise possibilitou perceber a importância da Estratégia Saúde da Família e dos Núcleos de Apoio à Estratégia Saúde da Família para a revitalização da Atenção Básica, e desta como local privilegiado para a realização das ações de alimentação e nutrição e de promoção da saúde. Essa relação apresenta potencialidades, mas também importantes desafios, como a necessidade de uma equipe interdisciplinar, para além da conformação atual das equipes de saúde da família, em número suficiente para atender a demanda e com qualificação para desenvolver ações de alimentação e nutrição, visando os princípios da universalidade, integralidade e equidade e a resolutividade dos serviços de saúde.

Palavras-chave: Alimentação. Nutrição em Saúde Pública. Atenção Primária à Saúde. Programa Saúde da Família. Promoção da Saúde. 


\section{Abstract}

Nutritional transition is one of the major challenges for public health policies because it demands a health care model guided by integrality and health promotion, especially in Primary Health Care. Food and nutrition are the basic requirements for health promotion and they enable the achievement of the potential of human development. This paper aims to reflect on the fields of food and nutrition in the context of Primary Care and Health Promotion, through descriptive and reflective methodology, reinforcing the need for a dialogue between these areas, through the interlocution between its challenges and potentials, in order to attain the principles of the National Health System. The analysis made it possible to realize the importance of the Family Health Strategy and Family Health Strategy Support for the revitalization of Primary Care, and how this place is privileged for conducting food and nutrition and health promotion activities. This relationship has potential, but also important challenges such as the need for an interdisciplinary team, which goes beyond the present conformation of family health teams, in sufficient numbers to meet the demand of skills and the development of food and nutrition actions, aiming for the principles of universality, integrality and fairness and the resoluteness of health services.

Key words: Feeding. Nutrition. Public Health. Primary Health Care. Family Health Program. Health Promotion.

\section{Introdução}

A transição nutricional, caracterizada pela coexistência de deficiências nutricionais, que têm diminuído, e das doenças crônicas não transmissíveis (DCNT), que têm aumentado, é um dos maiores desafios para as políticas públicas de saúde atualmente, pois demanda um modelo de atenção à saúde pautado na integralidade e uma abordagem voltada à promoção da saúde. ${ }^{1}$

Frente à necessidade de novos arranjos dos serviços, além da reorganização das ações de alimentação e nutrição desenvolvidas no sistema de saúde, a Atenção Básica (AB) e, em especial, a Estratégia Saúde da Família (ESF), são espaços privilegiados para ações de incentivo e apoio a hábitos de vida saudáveis, como aqueles relacionados à prática regular de atividade física e à alimentação. ${ }^{2,3} \mathrm{O}$ acesso à alimentação é um direito humano que se constituiu no próprio direito à vida, e a alimentação e a nutrição permitem alcançar o potencial de crescimento e de 
desenvolvimento humano com qualidade de vida e cidadania, conforme destaca a Política Nacional de Alimentação e Nutrição (PNAN). ${ }^{4}$

Esse também é um eixo fundamental da Política Nacional de Promoção da Saúde (PNPS), a qual também traz a importância do trabalho interdisciplinar e intersetorial, fundamentais na elevação da qualidade de vida da população. ${ }^{5}$ Além disso, a política ressalta que o apoio ao desenvolvimento de estudos referentes ao impacto de ações voltadas à alimentação na situação de saúde tem sido fundamental para que se possa alcançar a promoção da saúde. ${ }^{6}$

Nesse sentido, a PNAN reforça a necessidade de se manter atualizada uma agenda de prioridades de pesquisas em alimentação e nutrição, de interesse nacional e regional, pautada na agenda nacional de prioridades de pesquisa em saúde, com vistas a subsidiar os processos de tomada de decisão. ${ }^{4}$ Entretanto, persiste o desafio de desenvolver estudos e pesquisas que retratem as possibilidades de se trabalhar, de forma mais eficaz e abrangente, as ações de alimentação e nutrição nos serviços de saúde, principalmente no contexto da AB.

Assim, o presente artigo tem como objetivo refletir sobre os campos da Alimentação e Nutrição no contexto da Atenção Básica à Saúde e da Promoção da Saúde, por meio da metodologia descritivo-reflexiva baseada em pesquisa bibliográfica, reforçando a necessidade de um diálogo entre essas áreas, por meio da interlocução entre seus desafios e potencialidades, para que sejam alcançados, de forma eficaz, os princípios do Sistema Único de Saúde (SUS).

\section{A revitalização da atenção básica à saúde por meio da ESF e dos NASFs}

O histórico organizacional dos serviços de saúde orientados pela Atenção Básica à Saúde, expressão oficializada pelo governo brasileiro para o que, internacionalmente, se convencionou chamar de "Atenção Primária à Saúde", foi marcado por sucessivas reconstruções, até sua consolidação como uma política de reforma, frente à permanente crise dos sistemas de saúde contemporâneos. Desde a primeira metade do século XX, a AB vem, cada vez mais, exercendo papel fundamental na organização das ações dos sistemas de saúde de diversos países, sendo configurada como uma estratégia de reorientação do modelo assistencial. ${ }^{7,8}$

Sustentada no princípio da integralidade e definida como o primeiro nível de atenção à saúde, responsável pela articulação de ações de promoção da saúde, prevenção, tratamento e reabilitação de doenças e agravos, a AB tem sido compreendida como a porta de entrada preferencial ao sistema de saúde e abordagem que alicerça e determina o trabalho de todos os outros níveis. ${ }^{9,10}$ Assim, demanda uma intervenção ampla, para que se possa ter efeito positivo sobre a qualidade de vida da população, o que reflete a complexidade de sua atuação. ${ }^{11}$ 
No Brasil, a Política Nacional de Atenção Básica (PNAB), aprovada em 2006, em um contexto de descentralização e controle social da gestão, e revisada em 2011, reforçando o papel da AB na ordenação das Redes de Atenção à Saúde, tem a Estratégia Saúde da Família (ESF) como estratégia prioritária de reorganização. A ESF visa a expansão, qualificação e consolidação do primeiro nível da atenção, pois favorece uma reorientação do processo de trabalho, com maior potencial de aprofundar os princípios, diretrizes e fundamentos da $\mathrm{AB}$ e de ampliar a resolutividade e impacto na situação de saúde das pessoas e coletividades, de acordo com os princípios da universalidade, integralidade, equidade e descentralização, do SUS. ${ }^{2}$

A implantação da ESF ocasionou um importante avanço na AB brasileira. ${ }^{12}$ Contudo, apesar de ter sido acelerada nos municípios de pequeno porte, tem sido lenta nos grandes centros urbanos, em parte devido às questões de maior complexidade, relacionadas à concentração demográfica, ao grau elevado de exclusão no acesso aos serviços de saúde, aos agravos de saúde característicos das grandes cidades e metrópoles e à desarticulação e má distribuição da oferta consolidada na rede assistencial. ${ }^{13}$

Assim, a estruturação da rede de atendimento básico ainda representa um enorme desafio nos dias de hoje. A ausência de recursos humanos em saúde com capacitação técnica adequada e condições de trabalho atrativas constitui, ainda hoje, uma realidade na maioria dos municípios brasileiros, tornando-se grande obstáculo ao bom desempenho da gestão. ${ }^{9,14}$ Além disso, não basta redimensionar a formação para as equipes de saúde da família somente com maior número de cursos introdutórios ou horas de capacitação em treinamentos pontuais, mas redirecioná-la para um processo de ensino-aprendizagem continuado, problematizado e mediado, com a colaboração de diferentes profissionais. ${ }^{15}$

Visando apoiar e fortalecer a ESF na rede de serviços, para a ampliação da abrangência e do escopo das ações da AB, bem como sua resolutividade, o Ministério da Saúde criou, em 2008, o Núcleo de Apoio à Saúde da Família (NASF). Constituído por equipes compostas por profissionais de saúde de diferentes áreas de conhecimento, para atuarem em conjunto com os profissionais das equipes de saúde da família, através do matriciamento, os NASFs atuam, de forma interdisciplinar e intersetorial, prioritariamente com ações de educação permanente, com foco no território sob sua responsabilidade. ${ }^{16}$

\section{0 histórico organizacional e político das ações de alimentação e nutrição na atenção básica e na promoção da saúde}

Apesar da existência de registros de ações governamentais brasileiras no campo da alimentação e nutrição já na década de 1930, houve maior fortalecimento do tema na agenda nacional a partir 
da década de 1970, com a criação do Instituto Nacional de Alimentação e Nutrição (INAN) e, em especial, após a década de 1990, com a publicação de políticas e pesquisas na área.

A criação do INAN, em 1972, teve grande importância, pois este tinha a finalidade de assistir o governo na formulação da política nacional de alimentação e nutrição; elaborar, executar e avaliar o Programa Nacional de Alimentação e Nutrição (PRONAN); e funcionar como órgão central das atividades deste campo de conhecimento. O instituto também era responsável pela gestão do Programa de Nutrição em Saúde e do Programa de Combate às Carências Específicas. A extinção do INAN, em 1997, levou ao surgimento da Coordenação Geral da Política de Alimentação e Nutrição, atual Coordenação Geral de Alimentação e Nutrição, vinculada ao Ministério da Saúde e responsável pela elaboração da Política Nacional de Alimentação e Nutrição (PNAN). ${ }^{17}$

Na PNAN, aprovada em 1999, o Ministério da Saúde atestou seu compromisso, reconhecendo a alimentação e a nutrição como requisitos básicos para a promoção e proteção da saúde. A política também foi um marco para pesquisas no campo de nutrição científica, responsáveis por influenciar a mudança da concepção da relação entre alimentação e saúde, ${ }^{18}$ fortalecendo o reconhecimento da necessidade de investimentos na formulação, implementação e concretização de políticas de promoção, proteção e recuperação da saúde. ${ }^{8}$

Em 2011, a PNAN foi reeditada com o propósito de melhorar as condições de alimentação, nutrição e saúde, em busca da garantia da Segurança Alimentar e Nutricional da população brasileira, consolidando-se como referência aos novos desafios a serem enfrentados na área, dentro do SUS. ${ }^{4}$

Em articulação à PNAB e à PNAN, em 2006, foi elaborada a Política Nacional de Promoção da Saúde (PNPS), visando à criação de mecanismos que reduzissem as situações de vulnerabilidade, defendendo a equidade e incorporando a participação e o controle social na gestão das políticas públicas. A PNPS trouxe, como eixos estratégicos, a promoção da alimentação saudável com vistas à promoção de ações relativas à alimentação saudável, realização da intra e da intersetorialidade, implementação das ações de vigilância alimentar e nutricional e reorientação dos serviços de saúde, com ênfase na $\mathrm{AB} .^{6}$

Outro marco importante, já em 2009, foi a publicação da "Matriz de ações de alimentação e nutrição na Atenção Básica de Saúde”, publicada no ano seguinte à criação dos NASF. Veio com o objetivo de sistematizar e organizar as ações de alimentação e nutrição e do cuidado nutricional, para integrarem o rol de ações de saúde desenvolvidas no âmbito da AB, visando ao aperfeiçoamento da ação governamental, em especial no que tange às três políticas: PNAN, PNAB e PNPS. ${ }^{3}$

Atualmente, várias ações, na área de alimentação e nutrição vêm sendo implementadas pelo governo brasileiro. Jaime et al. ${ }^{19}$ destacam as ações de Vigilância Alimentar e Nutricional, com o uso do Sistema de Vigilância Alimentar e Nutricional; de Promoção da Saúde e da Alimentação 
Saudável, com a implementação do Guia Alimentar para a População Brasileira, da Estratégia Nacional para a Alimentação Complementar Saudável e do Programa Saúde na Escola; e de controle e prevenção da obesidade, doenças crônicas e deficiências de micronutrientes, com foco na anemia ferropriva e hipovitaminose A.

\section{Alimentação e nutrição na atenção básica à saúde: potencialidades e desafios}

Na busca por um modelo de atenção integral à saúde da população, fazem-se necessárias mudanças na organização das práticas e dos serviços, com transformações dos saberes e do processo de trabalho. ${ }^{20}$ Para isso, não se deve desvalorizar uma área do conhecimento em detrimento de outra, mas o contrário, partindo do pleno reconhecimento das especificidades que cada área possui, é preciso pensar em ações conjuntas, integradas e inter-relacionadas de profissionais de diferentes áreas, no intuito de superar a fragmentação do conhecimento ainda presente nos serviços de saúde oferecidos na AB. É importante que o profissional assuma seu papel de agente promotor da saúde, superando o paradigma de olhar a doença apresentada pelo usuário, e passando a contemplar o sujeito de forma plena, integral e contínua, associando saberes e práticas. ${ }^{18}$ Nesse sentido, tem-se avançado com programas como o Pró-Saúde e o PET-Saúde, que propõem reorientar a formação dos profissionais de saúde, perpassando as reformas curriculares e possibilitando maior integração ensino-serviço. ${ }^{21,22}$

É crucial, também, para a garantia da integralidade da atenção, avaliar os problemas encontrados e prover os recursos necessários, o que requer a integração dos serviços por meio de redes de atenção à saúde, sem precisar desrespeitar a interdependência entre atores e organizações, uma vez que nenhuma delas possui a totalidade dos recursos necessários para a resolução dos anseios e demandas de uma população. ${ }^{23}$ Assim, o acesso aos diferentes níveis de atenção à saúde deve ser universal, integral, contínuo e de qualidade, contribuindo para o desenvolvimento de relações de vínculo e responsabilização entre as equipes e os usuários. Também são importantes a valorização dos profissionais e a avaliação e acompanhamento dos resultados, bem como o estímulo à participação e controle social. ${ }^{3}$

No contexto da nutrição, os perfis epidemiológico e nutricional atuais têm composto um mosaico de agravos à saúde na população brasileira, levando a um despertar dos especialistas e das autoridades governamentais frente à necessidade de políticas que correspondam e supram as demandas advindas desse cenário. ${ }^{24}$ Diante disso, o desenvolvimento de ações de promoção das práticas alimentares saudáveis na $\mathrm{AB}$, em especial por meio da ESF, tem sido apontado como importante estratégia para enfrentar a nova realidade no campo da saúde. ${ }^{3,25,26}$

São notórios os avanços advindos dos esforços do Estado brasileiro, dentre os quais se destacam: a consolidação da PNAN; o aumento da oferta de ações de nutrição na AB, especialmente após 
implantação do NASF; a inclusão da temática alimentar e nutricional cada vez mais presente em estudos e publicações científicas; e o monitoramento das ações, por meio do Sistema de Vigilância Alimentar e Nutricional. Porém, para que as ações de alimentação e nutrição ofertadas possam suprir a demanda existente nos serviços de saúde, ainda existem nós críticos importantes.

Coutinho et al. ${ }^{1}$ mencionam a necessidade de fortalecer a rede de nutrição no SUS, institucionalmente, nas três esferas de governo, para que se possa implementar uma agenda única de nutrição, focada na promoção da alimentação saudável. Nesse sentido, ainda é preciso avançar rumo à consolidação, expansão e universalização das ações propostas para a AB no Brasil, como no acompanhamento da Vigilância Alimentar e Nutricional e do Programa Bolsa Família, no enfrentamento das deficiências de micronutrientes e na prevenção e controle das doenças crônicas. ${ }^{19}$

Há dez anos, Assis et al. ${ }^{5}$ destacaram que o objetivo de promover a saúde, especialmente no Brasil, não seria alcançado sem que ações efetivas, na área da vigilância e da assistência alimentar e nutricional, fossem implementadas de forma articulada a um sistema de vigilância à saúde. Além disso, os autores questionaram os possíveis rumos que uma reforma da $\mathrm{AB}$ teriam, sem a integração da nutrição e do nutricionista em sua prática. Possivelmente, a atenção à saúde continuaria centrada na velha hegemonia do conhecimento médico. Portanto, a reflexão sobre a inserção do nutricionista nas equipes da ESF se faz necessária.

Geus et al. ${ }^{27}$ enfatizam que essa inserção é fundamental para a promoção da saúde da população em todas as fases da vida, na abordagem dos aspectos de alimentação saudável, segurança alimentar, cidadania e direito humano à alimentação adequada. A ausência do nutricionista na equipe confronta-se com o princípio da integralidade das ações de saúde, já que este profissional é quem possui formação acadêmica para atuar na área de alimentação e nutrição dentro das comunidades e a abordagem do tema perpassa as ações de promoção e recuperação da saúde e prevenção e tratamento de doenças.

O profissional nutricionista ainda não faz parte das equipes básicas de saúde da família, porém pode exercer o matriciamento das ações de alimentação e nutrição nas equipes do NASF. ${ }^{28}$ Esse matriciamento representa uma potencialidade, mas traz consigo o desafio da apropriação das ações de alimentação e nutrição por outros profissionais de saúde, respeitando-se competências e atribuições específicas. Isso remete à importância da formação de profissionais aptos a colocar em prática as ações propostas para a AB. ${ }^{19}$ Entretanto, a implantação desses núcleos ainda se mostra incipiente, sendo necessário fortalecê-los e ampliá-los para facilitar o atendimento às demandas médico-sociais que, ainda hoje, ficam represadas na Unidade de Saúde da Família.15

De forma geral, cada NASF 1 deve realizar suas atividades vinculado a, no mínimo, oito e, no máximo, 15 equipes de Saúde da Família. ${ }^{2}$ Em 2012, de acordo com a Sala de Apoio à Gestão Estratégia, do Ministério da Saúde, havia 1.929 NASFs em funcionamento. No ano anterior, havia 1.564 equipes, com implantação dos NASFs em apenas 1.330 municípios brasileiros. ${ }^{29}$ 
O nutricionista é apenas um dos 20 profissionais do rol que podem compor os NASFs, sendo esta composição definida pelos gestores municipais. ${ }^{2}$ Essa flexibilidade na escolha dos profissionais visa permitir que as ações sejam planejadas de acordo com a realidade local, com base no território, constituindo, de fato, um planejamento ascendente. Isso representa uma grande potencialidade, sobretudo quando se consideram o modelo federativo brasileiro e a estruturação da rede de atenção à saúde, com a $\mathrm{AB}$ como ordenadora. Por outro lado, a condição de escolha também traz desafios que ainda devem ser superados, pois não garante que esse critério de prioridade seja utilizado, podendo a escolha e a contratação dos profissionais serem utilizadas para outros interesses, como interesses políticos, e até mesmo como "moeda de troca" de favores.

Também a promoção à saúde coloca-se como um desafio, no sentido de avançar na consolidação de um modelo intersetorial, com ações para além do espaço físico das Unidades Básicas de Saúde. A promoção traz consigo uma nova lógica de enfrentamento no processo de saúde-doença, considerando os determinantes sociais de saúde, que englobam aspectos sociais, econômicos, culturais, étnico-raciais, psicológicos e comportamentais. ${ }^{30}$

A intersetorialidade é uma das vertentes das ações de Alimentação e Nutrição na AB, com a integração das ações dos diferentes setores presentes no território, em especial, Educação e Assistência Social. Pode-se citar, como exemplo, o atendimento às famílias beneficiárias do Programa Bolsa Família. ${ }^{19}$ Como apontam Ferreira e Magalhães, ${ }^{31}$ a proposta de promoção traz consigo novas exigências em relação ao modelo de atenção e à formação acadêmica, com necessidade de romper a fragmentação tecnicista tradicional, e no que tange ao fortalecimento das parcerias e à inovação das práticas alimentares voltadas à construção da cidadania alimentar.

Por fim, outra questão a ser superada diz respeito ao direcionamento das pesquisas relacionadas à nutrição no âmbito da $\mathrm{AB}$ no Brasil. A condução de estudos que avaliem a prática profissional, as percepções e a educação permanente de profissionais de saúde da AB são de grande relevância. Apesar da produção na área ser crescente, os estudos relacionados à avaliação de programas de alimentação e nutrição, no geral, não tratam do impacto, mas apenas de sua implementação. É necessária ainda uma descentralização das pesquisas, bem como uma abrangência maior dos temas que envolvem a alimentação e nutrição na $\mathrm{AB} .{ }^{32}$

\section{Considerações finais}

Há décadas, a saúde deixou de ser vista apenas como a ausência de doença e seu conceito se tornou mais amplo, senda considerada um recurso para a vida e não como o objetivo de viver. ${ }^{33}$ Também passou a se pensar no bem-estar físico, social e psicológico. Dessa forma, não é possível pensar em AB e ESF sem pensar em ações de Promoção da Saúde, pois se perderia seu papel primordial, de proposição de um novo modelo de atenção integral à saúde, e se reforçaria aquele 
centrado na enfermidade. O processo de trabalho diferenciado da ESF, voltado ao contato com a realidade e à criação de vínculo com a comunidade, favorece a qualidade de vida da população e torna-se espaço privilegiado para a realização das ações de alimentação e nutrição, na perspectiva do Direito Humano à Alimentação Adequada.

Assim, as políticas de AB, alimentação e nutrição e promoção da saúde relacionam-se de forma interdependente e precisam ser trabalhadas de forma articulada, para que se possa implementar uma agenda única, voltada para a promoção de hábitos de vida saudáveis, considerando-se os determinantes sociais da saúde e a complexidade dos comportamentos humanos.

Para isso, a presença de uma equipe multi e interdisciplinar, para além da conformação atual das equipes de saúde da família, centradas, muitas vezes, no médico, é essencial. Todos os profissionais devem ser promotores da saúde e uma equipe ampliada na $\mathrm{AB}$, com outros saberes - com nutricionistas, profissionais de educação física, fonoaudiólogos, psicólogos, pedagogos, comunicólogos e tantos outros - traria grandes avanços rumo aos princípios da universalidade, da integralidade, da equidade e da resolutividade. E não basta somente ter os profissionais na rede, é preciso que seja em número suficiente para atender a demanda e realizar as ações que o SUS se propõe, o que se torna difícil com os profissionais inseridos apenas nas equipes do NASF, realizando prioritariamente o matriciamento, muitas vezes, de um número grande de equipes de saúde da família.

O matriciamento possui grande relevância na $\mathrm{AB}$, pois as ações de alimentação e nutrição e a promoção da alimentação saudável devem ser transversais, como parte da rotina de trabalho dos profissionais da equipe de saúde. Porém, ações mais específicas são muito relevantes e é importante que o contato dos usuários com o profissional nutricionista não se dê apenas depois da instalação das patologias, pois, nesse caso, seria a continuidade de um modelo curativista, pautado na doença, e o papel do nutricionista como promotor da saúde, diretamente, estaria em segundo plano ou, muitas vezes, nem acontecendo.

O nutricionista tem importante papel para subsidiar a população com informações relativas à Educação Alimentar e Nutricional. Porém, pensar uma alimentação saudável vai além das escolhas individuais. A alimentação é influenciada por questões relacionadas à agricultura, à educação, à economia, aos hábitos familiares e comunitários, à religião e a muitos outros fatores, objetivos e subjetivos, conscientes e inconscientes. Enfim, reflete os modos de andar a vida das pessoas e das comunidades. E, nesse aspecto, a presença do nutricionista na Atenção Básica se faz bastante importante, pois é esse o local mais próximo de onde as pessoas vivem e convivem.

É importante conhecer todo o contexto para que se possa agir, favorecendo-o. Para além da carência e da suplementação de um nutriente, por exemplo, é preciso conhecer toda uma cadeia que está por trás, desde a produção dos alimentos até o acesso por parte da comunidade. Esse trabalho deve ser feito pelos profissionais da equipe de saúde, e ter alguém com uma formação específica na área de alimentação e nutrição pode enriquecer ainda mais o trabalho. 
Além disso, é necessário que o trabalho seja feito em conjunto com a comunidade, que esta também possa participar ativamente dos processos de construção, com seus saberes, suas tradições e seus hábitos, favorecendo a sustentabilidade das ações. Há um movimento crescente para que os profissionais da Atenção Básica conheçam, de fato, a comunidade em que realizam seu trabalho e para que o Nutricionista realize ações além do atendimento nutricional de forma individualizada, sendo um articulador com o mercado produtivo, com os movimentos sociais, com os diversos setores, atuante nas arenas políticas.

Por fim, a formação e os processos de educação continuada e permanente dos profissionais de saúde precisam ser repensados, e, no contexto da nutrição, é imprescindível e estratégica a qualificação de gestores e trabalhadores de saúde para implementação de políticas, programas e ações voltados à atenção e vigilância alimentar e nutricional, promoção da saúde e da alimentação adequada e saudável e segurança alimentar e nutricional, para que se possa enfrentar os agravos e problemas decorrentes do atual quadro social, epidemiológico e nutricional brasileiro.

\section{Agradecimentos}

Ao Núcleo de Estudos de Saúde Pública, do Centro de Estudos Avançados Multidisciplinares, da Universidade de Brasília.

\section{Referências}

1. Coutinho JG, Gentil PC, Toral N. A desnutrição e obesidade no Brasil: o enfrentamento com base na agenda única da nutrição. Cad Saude Publica. 2008;24(supl. 2):332-40.

2. Brasil. Ministério da Saúde. Secretaria de Atenção à Saúde. Departamento de Atenção Básica. Política Nacional de Atenção Básica. Brasília: Ministério da Saúde; 2012.

3. Brasil. Ministério da Saúde. Secretaria de Atenção à Saúde. Departamento de Atenção Básica. Matriz de ações de alimentação e nutrição na atenção básica de saúde. Brasília: Ministério da Saúde; 2009.

4. Brasil. Ministério da Saúde. Secretaria de Atenção à Saúde. Departamento de Atenção Básica. Política Nacional de Alimentação e Nutrição. Brasília: Ministério da Saúde; 2012.

5. Assis AMO, et al. O Programa Saúde da Família: contribuições para uma reflexão sobre a inserção do nutricionista na equipe multidisciplinar. Rev Nutr. 2002;15(3):255-66.

6. Brasil. Ministério da Saúde. Secretaria de Vigilância em Saúde. Secretaria de Atenção à Saúde. Política Nacional de Promoção da Saúde. Brasília: Ministério da Saúde; 2006.

7. Conill EM. Ensaio histórico-conceitual sobre a Atenção Primária à Saúde: desafios para a organização de serviços básicos e da Estratégia Saúde da Família em centros urbanos no Brasil. Cad Saude Publica. 2008;24(supl. 1):7-27. 
8. Baptista TWF, Fausto MCR, Cunha MS. Análise da produção bibliográfica sobre atenção primária à saúde no Brasil em quatro periódicos selecionados. Physis. 2009;19(4):1007-28.

9. Sousa MF, Hamann EM. Programa Saúde da Família no Brasil: uma agenda incompleta? Ciênc saude coletiva. 2009;14(supl.1):1325-35.

10. Paim J, et al. The Brazilian health system: history, advances, and challenges. Lancet. 2011;377(9779):1778-97.

11. Starfield B. Atenção primária: equilíbrio entre necessidades de saúde, serviços e tecnologia. Brasília: Ministério da Saúde; 2002.

12. Escorel S, et al. O Programa de Saúde da Família e a construção de um novo modelo para a atenção básica no Brasil. Rev Panam de Salud Publica. 2007;21(2-3):164-76.

13. Sousa MF, et al. Gestão da atenção básica: redefinindo contextos e possibilidades. Divulg saude debate. 2000;21:7-14.

14. Besen CB, et al. A estratégia saúde da família como objeto de educação em saúde. Saude soc. 2007;16(1):57-68.

15. Kanno NP, Bellodi PL, Tess BH. Profissionais da Estratégia Saúde da Família diante de demandas médico-sociais: dificuldades e estratégias de enfrentamento. Saude soc. 2012;21(4):884-94.

16. Brasil. Ministério da Saúde. Secretaria de Atenção à Saúde. Departamento de Atenção Básica. Diretrizes do NASF: Núcleo de Apoio a Saúde da Família. Brasília: Ministério da Saúde; 2010.

17. Arruda BKG, Arruda IKG. Marcos referenciais da trajetória das políticas de alimentação e nutrição no Brasil. Rev Bras Saude Mater Infant 2007;7(3):319-26.

18. Silva DO, Recine EGIG, Queiroz EFO. Concepções de profissionais de saúde da atenção básica sobre a alimentação saudável no Distrito Federal, Brasil. Cad Saude Publica. 2002;18(5):1367-77.

19. Jaime PC, et al. Ações de alimentação e nutrição na atenção básica: a experiência de organização no Governo Brasileiro. Rev Nutr. 2011;24(6):809-24.

20. Favoreto CAO, Camargo Júnior KR. Alguns desafios conceituais e técnico-operacionais para o desenvolvimento do Programa de Saúde da Família como uma proposta transformadora do modelo assistencial. Physis. 2002;12(1):59-75.

21. Brasil. Lei n 11.180, de 23 de setembro de 2005. Institui o Programa de Educação Tutorial - PET e dá outras providências. Brasília; 2005.

22. Brasil. Portaria Interministerial no 1.802, de 26 de agosto de 2008. Institui o Programa de Educação pelo Trabalho para a Saúde - PET-Saúde. Brasília; 2008.

23. Shimizu HE, Rosales C. As práticas desenvolvidas no Programa Saúde da Família contribuem para transformar o modelo de atenção à saúde? Rev Bras Enferm. 2009;62(3):424-9.

24. Batista Filho M, Rissin A. A transição nutricional no Brasil: tendências regionais e temporais. Cad Saude Publica. 2003;19(supl. 1):181-91. 
25. Rizzolo AP. A promoção da alimentação saudável como instrumento de prevenção e combate ao sobrepeso e obesidade. Brasília: Ministério da Saúde; 2003. 15 p. Disponível em: http://www.saude. gov.br/alimentacao

26. Schmitiz BAS, et al. A escola promovendo hábitos alimentares saudáveis: uma proposta metodológica de capacitação para educadores e donos de cantina escolar. Cad Saude Publica. 2008;24(supl. 2):312-22.

27. Geus LMM, et al. A importância na inserção do nutricionista na Estratégia Saúde da Família. Ciênc saude coletiva. 2011;16(supl. 1):797-804.

28. Cervato-Mancuso AM, et al. A atuação do nutricionista na Atenção Básica à Saúde em um grande centro urbano. Ciênc saude coletiva. 2012;17(12):3289-300.

29. Brasil. Ministério da Saúde. Sala de Apoio à Gestão Estratégica. Disponível em: http://189.28.128.178/sage/

30. Buss PM, Pelegrini Filho A. A saúde e seus determinantes sociais. Physis. 2007;17(1):77-93.

31. Ferreira VA, Magalhães R. Nutrição e promoção da saúde: perspectivas atuais. Cad Saude Publica. 2007;23(7):1674-81.

32. Canella DS, Silva ACF, Jaime PC. Produção científica sobre nutrição no âmbito da Atenção Primária à Saúde no Brasil: uma revisão de literatura. Ciênc saude coletiva. 2013;18(2):297-308.

33. Brasil. Ministério da Saúde. Secretaria de Políticas de Saúde. Projeto promoção da saúde. As cartas de promoção da saúde. Brasília: Ministério da Saúde; 2002.

Recebido: 24/4/2013

Revisado: 29/7/2013

Aprovado: 02/8/2013 